\title{
Implications of telomerase reverse transcriptase in tumor metastasis
}

\author{
Yongkang Zou ${ }^{3}$, Yu-sheng Cong ${ }^{1,2} \mathcal{E}$ Junzhi Zhou ${ }^{1,2, *}$ \\ ${ }^{1}$ Key Laboratory of Aging and Cancer Biology of Zhejiang Province, Hangzhou Normal University, Hangzhou, Zhejiang $311121,{ }^{2}$ Institute \\ of Aging Research, Hangzhou Normal University School of Medicine, Hangzhou, Zhejiang 311121, ${ }^{3}$ Institute of Cancer Research, \\ Shenzhen Bay Laboratory, Shenzhen 518107, China
}

\begin{abstract}
Metastasis is the main culprit of the great majority of cancerrelated deaths. However, the complicated process of the invasion-metastasis cascade remains the least understood aspect of cancer biology. Telomerase plays a pivotal role in bypassing cellular senescence and sustaining the cancer progression by maintaining telomere homeostasis and genomic integrity. Telomerase reverse transcriptase (TERT) exerts a series of fundamental functions that are independent of its enzymatic cellular activity, including proliferation, inflammation, epithelia-mesenchymal transition (EMT), angiogenesis, DNA repair, and gene expression. Accumulating evidence indicates that TERT may facilitate most steps of the invasion-metastasis cascade. In this review, we summarize important advances that have revealed some of the mechanisms by which TERT facilitates tumor metastasis, providing an update on the non-canonical functions of telomerase beyond telomere maintaining. [BMB Reports 2020; 53(9): 458-465]
\end{abstract}

\section{INTRODUCTION}

Telomerase is a large ribonucleoprotein complex responsible for adding telomeric repeats to the $3^{\prime}$ ends of chromosomes, thereby alleviating the telomere attrition from each round of replication $(1,2)$. Telomerase is essentially composed of the TERT and telomerase RNA component (TR), which serves as a template for the TERT to synthesize telomeric repeats. hTR is ubiquitously expressed in both normal cells and cancer cells. Conversely, hTERT is repressed in normal cells, re-activated in $85-90 \%$ of cancer types, and closely associated with telomerase activity in cancers, suggesting that TERT is the determinant for telomerase activity, which makes telomerase an

*Corresponding author. Tel: +86-571-28861641; Fax: +86-571-2886 1715; E-mail: zhoujunzhi2013@163.com

https://doi.org/10.5483/BMBRep.2020.53.9.108

Received 22 May 2020, Revised 8 July 2020, Accepted 14 July 2020

Keywords: Cellular senescence, EMT, Metastasis, Telomerase, TERT attractive therapeutic target (3-6).

Tumor metastasis, termed the invasion-metastasis cascade, is a multi-step process that involves dissemination of cancer cells from primary tumors to distant organs and their subsequent seeding of new tumor colonies into foreign soil (7). The invasion-metastasis cascade is a succession of cell-biology events: (a) local invasion of primary tumor cells into the adjacent parenchyma through the surrounding extracellular matrix and stromal cell layers, (b) intravasation of invasive tumor cells into the circulatory system, (c) survival and dissemination during hematogenous transit through the vasculature, (d) arrest at distant organs, (e) extravasation of tumor cells from the microvasculature into the adjacent parenchyma of distant organ sites, (f) formation of micrometastase colonies in the parenchyma of distant tissues, and (g) growth of micrometastase colonies and establishment of a full-fledged tumor at the distant site (7). Metastatic disease is largely incurable because of it is complex and systemic and resists current therapeutic strategies, which results in more than $90 \%$ of deaths of cancer-associated patients (8). Recently, a growing number of evidence suggests that TERT may play a pivotal role in the invasion-metastasis cascade. Here, we highlight the functional regulation of TERT in each step of the tumor metastasis, which providing new insights for understanding tumor metastasis and developing new diagnostic and therapeutic strategies.

\section{TERT AND LOCAL INVASION}

Primary tumor cells breach the basement membrane (BM) and enter into the surrounding tumor-associated stroma, which leads to the first step in tumor metastasis. It has been demonstrated that metalloproteinases (MMPs), which disintegrate the extracellular matrix and promotes tumor cell invasion. Therefor, MMPs are key factors for determining the invasive potential of cells (9). It has been shown that hTERT associates with the expression of MMPs in many tumor entities. For example, knockdown hTERT in oral squamous-cell carcinoma decreases the MMP2 and MMP9 expression, thereby inhibiting invasiveness (10). Moreover, hTERT promotes the invasive ability of the cell by regulating MMP9 expression in a NF-кB-dependent

ISSN: 1976-670X (electronic edition)

Copyright (C) 2020 by the The Korean Society for Biochemistry and Molecular Biology

(c) This is an open-access article distributed under the terms of the Creative Commons Attribution Non-Commercial License (http://creativecommons.org/licenses/by-nc/4.0) which permits unrestricted non-commercial use, distribution, and reproduction in any medium, provided the original work is properly cited. 
manner independently of its catalytic activity in osteosarcoma and cervical carcinoma cell line, such as U2OS and HeLa (11). Importantly, Ghosh et al. revealed that TERT directly regulates NF-kB-dependent transcription $(11,12)$. To mention briefly,

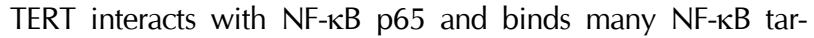
geting genes, such as IL6, IL8, and TNF $\alpha$, which are critical for inflammatory response (12). IL6 and TNF $\alpha$ play a central role in linking inflammation and cancer (13). It's worth noticing that IL-6 can act as an inducer of EMT in breast cancer cells (14). Ectopic expression of IL-6 in breast adenocarcinoma cells induces an EMT phenotype by upregulating vimentin, $\mathrm{N}$-cadherin, Snail and Twist. It's important to mention that TNF $\alpha$ enhances the invasive property of renal carcinoma cells by inducing EMT through Snail or ZEB1/ZEB2 $(15,16)$. EMT is believed to be an important step in the conversion of primary cancer cells into a migratory population capable of systemic metastasis (17-19). Thus, it seems that TERT contributes to the formation of migratory cell by inducing EMT in a NF- $\mathrm{KB}$-dependent manner. Interestingly, one study demonstrated that hTERT directly stimulates EMT of gastric cancer cells by occupying the promoter of vimentin in cooperation with $\beta$-catenin and in a telomere-independent manner (20). Collectively, hTERT promotes the invasiveness of cancer cells by inducing an extracellular protease and EMT program in a NF- $\mathrm{KB}$-dependent transcription manner. However, the detailed mechanisms by which TERT regulate NF-kB for primary tumor cells in local invasion remains to be revealed. TERT can use other extracellular proteases to remodel the ECM, thereby promoting the invasiveness of cells. It has been shown that cathepsins and heparanase can cleave the E-cadherin, a key cell-cell adhesion molecule, and degrade heparan sulfate proteoglycans (HSPG) secreted by ECM and BM, respectively, enabling primary tumor cells to cross the adjacent parenchyma (21-25). Importantly, Young et al. found that hTERT activates tumor invasiveness by upregulating cathepsin $\mathrm{D}$ via early growth response 1 (EGR-1) in oral squamous-cell carcinoma cells (22). Tang et al. found that hTERT expression is highly associated with an advanced TNM stage and lymphatic metastasis in gastric cancers. They further revealed that hTERT promotes gastric cancer-cell invasion and metastasis by cooperating with c-Myc to induce the expression of $\mathrm{Hpa}$ (23). These findings suggest that TERT may facilitate invasion of primary tumor cells via cathepsins and heparanase. However, this hypothesis needs more experimental support. Furthermore, whether TERT regulates the expression of cathepsins and heparanase dependent on its catalytic activity remains to be answered. Recently, studies demonstrated that TERT promote gastric cancer invasion by upregulating the expression of ITGB1 (Integrin $\beta 1$ ) adhesion molecules for cancer cells and the extracellular matrix (26, 27). The hTERT-MDM2 complex enhances the ubiquitin-mediated degradation of FOXO3a, alleviating FOXO3a-repressed ITGB1 expression, which eventually leads to the upregulation of ITGB1. Both hTERT and ITGB1 were highly expressed in the advanced tumor-node metastases (TNM) stage group and the lymphatic metastasis positive group. The group of higher expression of both hTERT and ITGB1 is linked to the worst survival rate in gastric-cancer patients. These findings suggest that TERT may contribute to tumor metastasis via ITGB1. Early studies showed that Integrin $\beta 1$ is a critical effector in promoting invasion by the primary tumor cells and cancer metastasis, which further supports that ITGB1 is a driver in tumor metastasis $(28,29)$.

Recently, Chen et al. showed that hTERT promotes the invasion of colorectal cancer cells by increasing chemokine CCL2 expression in collaboration with the $\beta$-catenin/TCF-4 complex. They further found that MDSCs accumulated in areas where hTERT and CCL2 were colocalized (30). Early studies revealed that primary tumor cells can use CCL2 to recruit MDSCs, regulatory T cells (TRegs) and tumor associate macrophage (TAM) to evade immune surveillance, which is a crucial step for preparing a metastatic journey to start the invasionmetastasis cascade (31-35). These results suggest that hTERT may also play a regulatory role in the recruitment of myeloid-derived suppressor cells (MDSCs) and evasion of tumor immunity.

\section{TERT AND INTRAVASATION}

Intravasation is local invasive cancer cells entering into the lumina of lymphatic or blood vessels to disseminate throughout the body before adhering to the endothelial cells lining the microvasculature (36). Tumor-associated microvasculature, generated by the process of angiogenesis, harbors the weak interactions between adjacent endothelial cells and the absence of extensive pericyte coverage, which are considered to facilitate intravasation (7). Therefore, the mechanics of intravasation are likely to be strongly influenced by angiogenesis. It has been well established that vascular endothelial growth factor (VEGF) is a key inducer in angiogenesis, which can stimulate tumor cells to form new blood vessels within their local microenvironment via neoangiogenesis (9). Importantly, it has been shown that hTERT induces VEGF gene expression independently of its telomerase reverse transcriptase activity, which provides a cornerstone for hTERT driving angiogenesis (37). Liu et al. reported that hTERT induces VEGF gene expression by occupying the VEGF promoter in cooperation with Sp1 (38). They further found that TERT promotes the vascular tube formation and deficiency of TERT-compromised tumor growth and vascular development. Furthermore, hTERT expression is positively correlated with VEGF in patient samples. These findings suggest that TERT may facilitate the intravasation by inducing angiogenesis. It has been previously reported that VEGF can induce hTERT and telomerase activity $(39,40)$. There may be a positive regulatory feedback circuit between hTERT and VEGF that has a collaborative role in tumorigenesis and cancer progression. Besides VEGF, sufficient studies showed that MMPs are involved in angiogenesis-dependent intravasation and metastasis, and that MMPs can directly or 
indirectly affect the VEGF-mediated development of angiogenic vasculature. These findings suggest that TERT may also contribute to the induction of intravasation and metastasis-sustaining neo-vasculature via MMPs. A growing number of studies showed that TGF- $\beta$ promote intravasation $(41,42)$. In order to identify the role of mTert in prostate-cancer progression, Ding et al. compared an mTert-deficiency mouse (G3/4-LSL- mTert $^{-1-}$ Pten/p53) with an mTert knock-in mouse (G3/4-LSL- mTert $^{+/+}$Pten/p53) and found that the TGF- $\beta / S m a d 4$ pathway is required for mTERT-mediated bone metastasis (43). It seems that mTert acts as an active driver for tumor metastasis in a telomerebased crisis. We can assume that the TGF- $\beta / S \operatorname{Smad} 4$ pathway may be implicated in the process of intravasation. However, the mechanisms by which mTERT regulate TGF- $\beta$ in bone metastasis need to be discovered.

\section{TERT AND CIRCULATING TUMOR CELLS' (CTCS) SURVIVAL IN CIRCULATION}

The bloodstream represents a hostile environment for CTCs, exposing various cellular stresses that include oxidative stress and endoplasmic reticulum (ER) stress $(7,44,45)$. It has been found by using animal models (46) that less than $0.02 \%$ of tumor cells in circulation can survive to form metastasis. To survive and disseminate in the circulation, CTCs not only induce survival-related pathways and the EMT program, but also interact with bioactive factors and stroma cells to facilitate their passage to extravasation at distant sites $(8,44)$. Tumor cells and tumor-associated stromal cells secrete abundant reactive oxygen species (ROS) into the tumor microenvironment (47). ROS exerts an important role in cellular activities, including genomic instability, cell death, and cellular senescence (48). CTCs use multiple pathways to adapt to the increased ROS, including modification of metabolism and up-regulation of $\beta$-hemoglobin $(49,50)$. More recently, evidence showed that oxidative stress inhibits distant metastasis $(49,51)$. To mention briefly, several studies demonstrated that TERT alleviates the production of ROS, improves mitochondrial function, and inhibits ROS-induced apoptosis in cancer cells (52-54). Moreover, CTCs are also exposed to various stress stimuli that activate the unfolded protein response (UPR), a homeostatic pathway, that is mainly composed of the IRE $1 \alpha$-xbp1, ATF6, and PERK-eIF2 $\alpha$ pathways and orchestrates several events to restore the ER folding capacity or can induce cell death when restoration fails $(47,55)$. Importantly, we previous reported that ER stress promotes hTERT expression in both human cancer cells and murine neural cells, which protects cells from ER stress-induced cell death (56). Moreover, overexpression of hTERT reduces ER stress-induced cell death, which is independent of catalytically active enzymes or DNA damage signaling, whereas depletion of hTERT sensitizes cells to undergo apoptosis under ER stress. Additionally, $\mathrm{Yu}$ et al. demonstrated that CTCs of breast tumors exhibit combinations of epithelial and mesenchymal traits, suggesting an important role for EMT in the blood-borne dissemination of human breast cancer (57). It seems reasonable that TERT may protect CTCs from oxidative stress or ER stress. So far, the mechanisms by which oxidative stress or ER stress regulates the CTCS survival are largely unknown. As mentioned above, there are less than $0.02 \%$ of tumor cells that can survive in circulating blood, which leads to a big bottleneck in collecting enough CTCs.

\section{TERT AND EXTRAVASATION}

CTCs may cross from vessel lumina into the tissue parenchyma by traversing the endothelial cell and pericyte layers, which is considered as extravasation (58). Accordingly, CTCs secret a series of factors to affect the microenvironments and promote vascular hyperpermeability, which leads to tumor cells across the barriers to extravasation in tissues (58). For example, it has been demonstrated that VEGF promotes the process of extravasation by inducing pulmonary hyperpermeability, which was prior to the arrival of CTCs in the lungs $(59,60)$. Moreover, MMPs facilitate the extravasation of breast tumor cells by disrupting pulmonary vascular endothelial cell-cell junctions $(42,61)$. Given that TERT can induce the expression of MMPs and VEGF, these findings suggest that TERT may contribute to the process of extravasation during metastasis. VEGF and MMPs may promote the processes of both intravasation and extravasation, suggesting that certain traits which were favorable previously in the process of tumor invasion also prove useful at the later steps in the invasion-metastasis cascade. However, the mechanisms by which TERT regulates the extravasation need to be addressed. Recently, studies have demonstrated that CCL2 promotes the extravasation and subsequent metastatic growth by directly increasing the vascular permeability in lung parenchyma $(58,62)$. Moreover, inflammatory monocytes facilitate the extravasation of breast carcinoma cells via a CCL2-dependent mechanism (62). It has been reported that hTERT promotes invasion and migration of colon- cancer cells by upregulating expression of CCL2 (30). Therefore, we speculate that TERT may contribute to the extravasation via CCL2. Taken together, TERT could induce VEGF, CCL2, and MMPs expression and enhance the vascular hyperpermeability, thereby promoting the intravasation. However, the detailed mechanisms by which TERT regulates the intravasation remain to be found.

\section{IMPLICATIONS OF TERT IN MICROMETASTASIS FORMATION AND MACROMETASTATIC GROWTH}

Cancer cells extravasate into the parenchyma of target tissues and start colonization after CTCs are arrested in capillaries at distant sites. Disseminated tumor cells (DTCs) land in a new tissue microenvironment, where they lack the familiar stromal cells, growth factors, cytokines, and ECM constituents that had been present in the primary tumor site (7). Hence, most DTCs 
arrive at the metastatic site as solitary tumor cells and have to resume growth to develop a new colony and establish a micrometastasis. Otherwise, they will die or enter dormancy (63). Early studies demonstrated that the ratio between the p38 mitogen-activated protein kinase (p38) and extracellular signalregulated kinase1/2 (ERK1/2) determines whether DTCs enter proliferation or dormancy (63-65). The activities of p38 and ERK are regulated by altered intrinsic signaling pathways and external dormancy-inducing signals (65-72). Of note, the property of tumor initiation in cancer stem cells (CSCs) is a prerequisite for successfully forming a metastatic colony (7). Interestingly, Okamoto et al. revealed that hTERT forms a complex with nucleolar GTP-binding protein nucleostemin (GNL3L/NS) and BRG1, which act in a telomere-independent manner to maintain the state of tumor-initiating cells or cancer stem cells (73). Thus, we speculate that TERT may drive DTCs in the status of cancer stem cells, which contributes to the formation of metastastic colonies. Moreover, some studies have demonstrated that external dormancy-inducing signals, including bone morphogenetic protein (BMP) ligand 7 (BMP7) and TGF- $\beta$, induce dormancy of tumor cells $(65,69)$. Importantly, Lucy et al. revealed that BMP7 negatively regulates telomere maintenance by suppressing hTERT, inducing cervical tumor-growth arrest (74). Li et al. found that TGF- $\beta$ suppresses TERT expression by Smad3 interacting with c-Myc and TERT promoter (75). Given that BMP7, TGF- $\beta$, and hTERT are important for underpinning cancer stem-cells renewal and proliferation, we speculate that the functional regulation of hTERT by BMP7 or TGF- $\beta$ may take an important role in establishing a balance between tumor dormancy and metastatic colonies' growth. Interestingly, Xu's lab reported that hTERT promotes in vivo tumorigenesis and metastastic colonies of gastric-cancer cells (20). It's the first direct evidence to support the assumption that hTERT facilitates the formation of metastastic colonies. The detailed mechanisms by which TERT maintains the dormancy of DTCs and promotes micrometastasis remain to be discovered.

\section{IMPLICATIONS OF TERT PROMOTER MUTATION IN TUMOR METASTASIS}

The TERT promoter hotspot mutations (C228T and C250T) have been widely investigated and identified as driver mutations with different frequencies from undetectable to $85 \%$ in various human cancer types $(76,77)$. Clinically, tumor samples carrying TERT promoter mutations promote the expression of TERT mRNA and telomerase activity compared to those having a wild-type promoter (78). Through experimentation, the introduction of either $-124 \mathrm{C}>\mathrm{T}$ or $-146 \mathrm{C}>\mathrm{T}$ into the TERT promoter reporter significantly increases the promoter activity $(77,79)$. It has been reported that GA-binding proteins were specifically recruited to the TERT mutant promoter compared to the wild-type TERT promoter, which enhances TERT transcription and telomerase activity (79). Therefore, it has been considered that the interaction between GA-binding proteins and the TERT promoter mutations forms a novel mechanism for telomerase activation in malignant transformation. It's interesting that accumulating evidence showed that TERT promoter mutations associate tumor metastasis with several cancer types, including melanoma, thyroid cancer, urothelial carcinomas, and glioblastomas. First, it has been reported that a higher frequency of TERT promoter mutations was observed in metastatic tissues than in primary melanomas. These mutations were associated with shorter survival or short disease-free survival in melanomas, suggesting the functional role of TERT promoter mutations in the metastatic process of melanoma cells (80). Second, it has been demonstrated that TERT promoter mutations are associated with distant metastasis, worse prognostic features, poor survival, and worse response to treatment in thyroid cancer (81-86). Third, evidence showed that TERT promoter mutations are related to distant metastases in glioblastomas and urothelial carcinomas (87). It's worth mentioning that the simultaneous mutation of BRAF/RAS and TERT promoter leads a high aggressiveness in thyroid cancer, melanoma, and lung cancer with different mechanisms being identified. For example, it has been proposed KRAS activates the expression of TERT by promoting the activity of RAS/MEK pathway, which leads aggressiveness of lung cancer cells (88). Moreover, Liu et al. demonstrated a novel mechanism for the activation of mutant TERT by the BRAF V600E/MAP kinase pathway through FOS/GABP in human cancer $(89,90)$. In summary, TERT promoter mutations that are implicated in the tumor metastasis and mechanisms by which TERT promoter mutations regulate the tumor metastasis are largely unidentified.

\section{CONCLUDING REMARKS}

In the past few years, sufficient evidence indicates that TERT has non-canonical functions beyond telomere maintenance in several important cellular processes, including cell proliferation, inflammation, apoptosis, EMT, cell adhesion and migration, angiogenesis, DNA damage response, and transcriptional regulation of target genes $(5,91,92)$. As summarized in this review, accumulating studies have revealed that TERT may facilitate most steps of invasion-metastasis cascade as summarized in Fig. 1 including:

(1) Promotes local invasion of primary cancer cells by using different factors and cellular programs, mainly including NF-kB, MMPs, EMT, and ITGB1.

(2) Promotes the intravasation through VEGF and TGF- $\beta$.

(3) Promotes CTCs survival by using an EMT program or anti-oxidative stress or ER stress-induced apoptosis.

(4) Promotes the extravasation through tumor-secreted VEGF, MMPs, and CCL2.

(5) Promotes micrometastasis formation and macrometastatic growth, in which EMT, hTERT/ GNL3L/NS/BRG1 complex, and external dormancy-inducing signals (such as, BMP7 and TGF- $\beta$ ) are involved. 


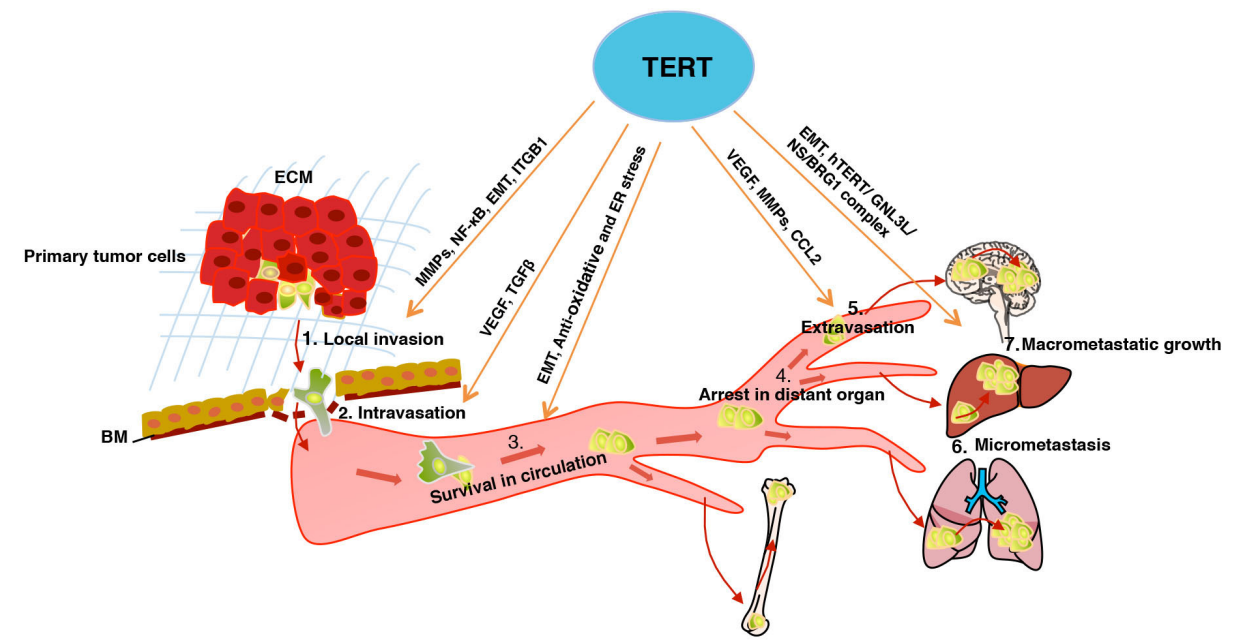

Fig. 1. TERT facilitates the invasion-metastasis cascade. TERT promotes local invasion via MMPs, NF-kB, EMT, and ITGB1. TERT promotes intravasation via TGF- $\beta$ and VEGF. TERT promotes CTCs survival in circulation via EMT and anti-oxidative stress and ER stress-induced apoptosis. TERT promotes extravasation via VEGF, MMPs, and CCL2. TERT promotes the formation of micrometastasis and the macrometastatic growth partially through EMT and hTERT/ GNL3LNSS/BRG1 complex. The actions of TERT implicated in tumor metastasis are described in the text. ECM, extracellular matrix. BM, basement membrane.

TERT may facilitate the invasion metastasis cascade, which suggests that TERT is a new diagnostic and attractive therapeutic target in tumor metastasis. In line with this hypothesis, several approaches have been adopted, including oligonucleotides (e.g., GRN163L), small-molecule inhibitors (e.g., BIBR1532), and anti-telomerase immune-therapeutics (GRVAC1, GV1001 and Vx001), to selectively induce apoptosis and cell death in cancer cells (93). GRN163L can efficiently target glioblastoma tumor-initiating cells, which leads to a decreased proliferation and tumor growth (94). Similarly, clinical trial results demonstrated that a vaccine (GV1001, hTERT peptide) is a promising as a telomerase-targeting vaccine and can stimulate $\mathrm{CD} 4^{+}$and $\mathrm{CD}^{+}$responses in telomerase-positive tumors, showing minimal effects on normal cells and no autoimmunity (93). However, metastatic disease is complex and systemic and resists most of current therapeutic strategies, which are closely affected by the tumor microenvironment, which plays a vital role in determining the success of therapeutic vaccination, but the mechanisms by which TERT is employed in the metastatic microenvironment are poorly understood. Thus, it's urgent to arrive at a precise understanding of the contribution of TERT in each step of the invasion-metastasis cascade.

It has been extensively studied how TERT promotes invasion by cancer cells. However, the detailed functional aspects and mechanisms by which TERT regulates the local invasion of primary tumor cells remain to be identified. Importantly, studies have shown that preparation for a metastatic journey, which is involved in evasion of tumor immunity and pre-metastatic niche, is a crucial step for primary tumors to start the invasion-metastasis cascade $(31,32)$. More questions need to be answered in the future:

(1) Whether TERT regulates the functional aspects of immune cells to affect the tumor invasion, including TAMs, TANs, MDSCs, Treg, and B cells.

(2) Whether TERT regulates the cytotoxicity of NK cells and CD8 T cells.

(3) Whether TERT promoter mutations (C228T and C250T) regulate the immune cells and the cytotoxicity of NK cells and CD8 T cells $(76,77)$.

Metastatic colonization may require several years or decades to complete, which provides a key therapeutic window for targeting metastatic colonization (95). Targeting CSCs may alleviate metastatic colonization (7). Importantly, several studies have suggested that TERT takes an important role in maintaining the state of CSCs and dormancy of DTCs via the TERTGNL3L-BRG1 complex and external dormancy-inducing signals $(65,69,73)$. However, more questions need to be answered:

(1) Whether both TERT and the TERT-GNL3L-BRG1 complex regulate the dormancy of DTCs.

(2) Mechanisms by which TERT regulates the external dormancyinducing signals, including BMP7 and TGF $\beta$.

(3) The detailed mechanism by which TERT promotes metastastic colonies of gastric cancer cells (20).

\section{ACKNOWLEDGEMENTS}

This work was supported by Hangzhou Normal University [grant number 4125C5021920462). 


\section{CONFLICTS OF INTEREST}

The authors have no conflicting interests.

\section{REFERENCES}

1. Finkel T, Serrano M and Blasco MA (2007) The common biology of cancer and ageing. Nature 448, 767-774

2. Palm W and de Lange T (2008) How shelterin protects mammalian telomeres. Annu Rev Genet 42, 301-334

3. Arndt GM and MacKenzie KL (2016) New prospects for targeting telomerase beyond the telomere. Nat Rev Cancer 16, 508-524

4. Yi X, Tesmer VM, Savre-Train I, Shay JW and Wright WE (1999) Both transcriptional and posttranscriptional mechanisms regulate human telomerase template RNA levels. Mol Cell Biol 19, 3989-3997

5. Cong Y and Shay JW (2008) Actions of human telomerase beyond telomeres. Cell Res 18, 725-732

6. Cong YS, Wright WE and Shay JW (2002) Human telomerase and its regulation. Microbiol Mol Biol Rev 66, 407-425, table of contents

7. Lambert AW, Pattabiraman DR and Weinberg RA (2017) Emerging Biological Principles of Metastasis. Cell 168, 670-691

8. Wan L, Pantel K and Kang Y (2013) Tumor metastasis: moving new biological insights into the clinic. Nat Med $19,1450-1464$

9. Hanahan D and Weinberg RA (2011) Hallmarks of cancer: the next generation. Cell 144, 646-674

10. Park YJ, Kim EK, Moon S, Hong DP, Bae JY and Kim J (2014) Human telomerase reverse transcriptase is a promising target for cancer inhibition in squamous cell carcinomas. Anticancer Res 34, 6389-6395

11. Ding D, Xi P, Zhou J, Wang M and Cong YS (2013) Human telomerase reverse transcriptase regulates MMP expression independently of telomerase activity via NFkappaB-dependent transcription. FASEB J 27, 4375-4383

12. Ghosh A, Saginc G, Leow SC et al (2012) Telomerase directly regulates NF-kappaB-dependent transcription. Nat Cell Biol 14, 1270-1281

13. Taniguchi K and Karin M (2018) NF-kappaB, inflammation, immunity and cancer: coming of age. Nat Rev Immunol 18, 309-324

14. Sullivan NJ, Sasser AK, Axel AE et al (2009) Interleukin-6 induces an epithelial-mesenchymal transition phenotype in human breast cancer cells. Oncogene 28, 2940-2947

15. Chuang MJ, Sun KH, Tang SJ et al (2008) Tumor-derived tumor necrosis factor-alpha promotes progression and epithelial-mesenchymal transition in renal cell carcinoma cells. Cancer Sci 99, 905-913

16. Chua HL, Bhat-Nakshatri $P$, Clare SE, Morimiya A, Badve $\mathrm{S}$ and Nakshatri H (2007) NF-kappaB represses E-cadherin expression and enhances epithelial to mesenchymal transition of mammary epithelial cells: potential involvement of ZEB-1 and ZEB-2. Oncogene 26, 711-724

17. Pastushenko I and Blanpain C (2019) EMT Transition States during Tumor Progression and Metastasis. Trends Cell Biol 29, 212-226
18. Thiery JP, Acloque $H$, Huang RY and Nieto MA (2009) Epithelial-mesenchymal transitions in development and disease. Cell 139, 871-890

19. Tam WL and Weinberg RA (2013) The epigenetics of epithelial-mesenchymal plasticity in cancer. Nat Med 19, 1438-1449

20. Liu Z, Li Q, Li K et al (2013) Telomerase reverse transcriptase promotes epithelial-mesenchymal transition and stem cell-like traits in cancer cells. Oncogene 32, 4203-4213

21. Vlodavsky I, Ilan N, Naggi A and Casu B (2007) Heparanase: structure, biological functions, and inhibition by heparinderived mimetics of heparan sulfate. Curr Pharm Des 13, 2057-2073

22. Park YJ, Kim EK, Bae JY, Moon S and Kim J (2016) Human telomerase reverse transcriptase (hTERT) promotes cancer invasion by modulating cathepsin $\mathrm{D}$ via early growth response (EGR)-1. Cancer Lett 370, 222-231

23. Tang B, Xie R, Qin $Y$ et al (2016) Human telomerase reverse transcriptase (hTERT) promotes gastric cancer invasion through cooperating with c-Myc to upregulate heparanase expression. Oncotarget 7, 11364-11379

24. Gocheva V, Zeng W, Ke D et al (2006) Distinct roles for cysteine cathepsin genes in multistage tumorigenesis. Genes Dev 20, 543-556

25. Turk V, Stoka V, Vasiljeva O et al (2012) Cysteine cathepsins: from structure, function and regulation to new frontiers. Biochim Biophys Acta 1824, 68-88

26. Hu C, Ni Z, Li BS et al (2017) hTERT promotes the invasion of gastric cancer cells by enhancing FOXO3a ubiquitination and subsequent ITGB1 upregulation. Gut 66, 31-42

27. He B, Xiao YF, Tang B et al (2016) hTERT mediates gastric cancer metastasis partially through the indirect targeting of ITGB1 by microRNA-29a. Sci Rep 6, 21955

28. Chen MB, Lamar JM, Li R, Hynes RO and Kamm RD (2016) Elucidation of the Roles of Tumor Integrin beta1 in the Extravasation Stage of the Metastasis Cascade. Cancer Res 76, 2513-2524

29. Xu Z, Zou L, Ma G et al (2017) Integrin beta1 is a critical effector in promoting metastasis and chemo-resistance of esophageal squamous cell carcinoma. Am J Cancer Res 7, 531-542

30. Chen S, Yang L, Dong H and Guo H (2019) Human telomerase reverse transcriptase recruits the beta-catenin/ TCF-4 complex to transactivate chemokine (C-C motif) ligand 2 expression in colorectal cancer. Biomed Pharmacother 112, 108700

31. Kitamura T, Qian BZ and Pollard JW (2015) Immune cell promotion of metastasis. Nat Rev Immunol 15, 73-86

32. Peinado H, Zhang H, Matei IR et al (2017) Pre-metastatic niches: organ-specific homes for metastases. Nat Rev Cancer 17, 302-317

33. Dirat B, Bochet L, Dabek M et al (2011) Cancer-associated adipocytes exhibit an activated phenotype and contribute to breast cancer invasion. Cancer Res 71, 2455-2465

34. Karnoub AE, Dash AB, Vo AP et al (2007) Mesenchymal stem cells within tumour stroma promote breast cancer metastasis. Nature 449, 557-563

35. Wyckoff J, Wang W, Lin EY et al (2004) A paracrine loop 
between tumor cells and macrophages is required for tumor cell migration in mammary tumors. Cancer Res 64, 7022-7029

36. Obenauf AC and Massague J (2015) Surviving at a Distance: Organ-Specific Metastasis. Trends Cancer 1, 76-91

37. Zhou L, Zheng D, Wang M and Cong YS (2009) Telomerase reverse transcriptase activates the expression of vascular endothelial growth factor independent of telomerase activity. Biochem Biophys Res Commun 386, 739743

38. Liu N, Ding D, Hao W et al (2016) hTERT promotes tumor angiogenesis by activating VEGF via interactions with the Sp1 transcription factor. Nucleic Acids Res 44, 8693-8703

39. Bermudez $Y$, Yang $H$, Saunders BO, Cheng JQ, Nicosia SV and Kruk PA (2007) VEGF- and LPA-induced telomerase in human ovarian cancer cells is Sp1-dependent. Gynecol Oncol 106, 526-537

40. Zaccagnini G, Gaetano C, Della Pietra L et al (2005) Telomerase mediates vascular endothelial growth factordependent responsiveness in a rat model of hind limb ischemia. J Biol Chem 280, 14790-14798

41. Giampieri S, Manning C, Hooper S, Jones L, Hill CS and Sahai E (2009) Localized and reversible TGFbeta signalling switches breast cancer cells from cohesive to single cell motility. Nat Cell Biol 11, 1287-1296

42. Padua D, Zhang XH, Wang Q et al (2008) TGFbeta primes breast tumors for lung metastasis seeding through angiopoietin-like 4. Cell 133, 66-77

43. Ding Z, Wu CJ, Jaskelioff $M$ et al (2012) Telomerase reactivation following telomere dysfunction yields murine prostate tumors with bone metastases. Cell 148, 896-907

44. Massague J and Obenauf AC (2016) Metastatic colonization by circulating tumour cells. Nature 529, 298-306

45. Senft D and Ronai ZA (2016) Adaptive Stress Responses During Tumor Metastasis and Dormancy. Trends Cancer 2, 429-442

46. Chambers AF, Groom AC and MacDonald IC (2002) Dissemination and growth of cancer cells in metastatic sites. Nat Rev Cancer 2, 563-572

47. Senft D and Ronai ZE (2016) Adaptive Stress Responses During Tumor Metastasis and Dormancy. Trends Cancer $2,429-442$

48. Gorrini C, Harris IS and Mak TW (2013) Modulation of oxidative stress as an anticancer strategy. Nat Rev Drug Discov 12, 931-947

49. Piskounova E, Agathocleous M, Murphy MM et al (2015) Oxidative stress inhibits distant metastasis by human melanoma cells. Nature 527, 186-191

50. Zheng Y, Miyamoto DT, Wittner BS et al (2017) Expression of beta-globin by cancer cells promotes cell survival during blood-borne dissemination. Nat Commun 8, 14344

51. Le Gal K, Ibrahim MX, Wiel C et al (2015) Antioxidants can increase melanoma metastasis in mice. Sci Transl Med 7, 308re308

52. Ahmed S, Passos JF, Birket MJ et al (2008) Telomerase does not counteract telomere shortening but protects mitochondrial function under oxidative stress. J Cell Sci $121,1046-1053$
53. Haendeler J, Drose S, Buchner N et al (2009) Mitochondrial telomerase reverse transcriptase binds to and protects mitochondrial DNA and function from damage. Arterioscler Thromb Vasc Biol 29, 929-935

54. Singhapol C, Pal D, Czapiewski R, Porika M, Nelson G and Saretzki GC (2013) Mitochondrial telomerase protects cancer cells from nuclear DNA damage and apoptosis. PLoS One 8, e52989

55. Hetz C (2012) The unfolded protein response: controlling cell fate decisions under ER stress and beyond. Nat Rev Mol Cell Biol 13, 89-102

56. Zhou J, Mao B, Zhou Q et al (2014) Endoplasmic reticulum stress activates telomerase. Aging Cell 13, 197-200

57. Yu M, Bardia A, Wittner BS et al (2013) Circulating breast tumor cells exhibit dynamic changes in epithelial and mesenchymal composition. Science 339, 580-584

58. Valastyan S and Weinberg RA (2011) Tumor metastasis: molecular insights and evolving paradigms. Cell 147, 275292

59. Huang Y, Song N, Ding Y et al (2009) Pulmonary vascular destabilization in the premetastatic phase facilitates lung metastasis. Cancer Res 69, 7529-7537

60. Weis S, Cui J, Barnes L and Cheresh D (2004) Endothelial barrier disruption by VEGF-mediated Src activity potentiates tumor cell extravasation and metastasis. J Cell Biol 167, 223-229

61. Gupta GP, Perk J, Acharyya S et al (2007) ID genes mediate tumor reinitiation during breast cancer lung metastasis. Proc Natl Acad Sci U S A 104, 19506-19511

62. Qian BZ, Li J, Zhang H et al (2011) CCL2 recruits inflammatory monocytes to facilitate breast-tumour metastasis. Nature 475, 222-225

63. Pantel K and Brakenhoff RH (2004) Dissecting the metastatic cascade. Nat Rev Cancer 4, 448-456

64. Aguirre-Ghiso JA, Estrada Y, Liu D and Ossowski L (2003) ERK(MAPK) activity as a determinant of tumor growth and dormancy; regulation by p38(SAPK). Cancer Res 63, 16841695

65. Yu-Lee LY, Yu G, Lee YC et al (2018) Osteoblast-Secreted Factors Mediate Dormancy of Metastatic Prostate Cancer in the Bone via Activation of the TGFbetaRIII-p38MAPKpS249/T252RB Pathway. Cancer Res 78, 2911-2924

66. Aguirre Ghiso JA, Kovalski K and Ossowski L (1999) Tumor dormancy induced by downregulation of urokinase receptor in human carcinoma involves integrin and MAPK signaling. J Cell Biol 147, 89-104

67. Shibue T and Weinberg RA (2009) Integrin beta1-focal adhesion kinase signaling directs the proliferation of metastatic cancer cells disseminated in the lungs. Proc Natl Acad Sci U S A 106, 10290-10295

68. Douma S, Van Laar T, Zevenhoven J, Meuwissen R, Van Garderen E and Peeper DS (2004) Suppression of anoikis and induction of metastasis by the neurotrophic receptor TrkB. Nature 430, 1034-1039

69. Kobayashi A, Okuda H, Xing F et al (2011) Bone morphogenetic protein 7 in dormancy and metastasis of prostate cancer stem-like cells in bone. J Exp Med 208, 2641-2655

70. Gao H, Chakraborty G, Lee-Lim AP et al (2012) The BMP inhibitor Coco reactivates breast cancer cells at lung 
metastatic sites. Cell 150, 764-779

71. Shiozawa $\mathrm{Y}$, Havens $\mathrm{AM}$, Pienta $\mathrm{KJ}$ and Taichman RS (2008) The bone marrow niche: habitat to hematopoietic and mesenchymal stem cells, and unwitting host to molecular parasites. Leukemia 22, 941-950

72. Zhang XH, Wang Q, Gerald W et al (2009) Latent bone metastasis in breast cancer tied to Src-dependent survival signals. Cancer Cell 16, 67-78

73. Okamoto N, Yasukawa M, Nguyen C et al (2011) Maintenance of tumor initiating cells of defined genetic composition by nucleostemin. Proc Natl Acad Sci U S A 108, 20388-20393

74. Cassar L, Li H, Pinto AR, Nicholls C, Bayne S and Liu JP (2008) Bone morphogenetic protein-7 inhibits telomerase activity, telomere maintenance, and cervical tumor growth. Cancer Res 68, 9157-9166

75. Li H, Xu D, Li J, Berndt MC and Liu JP (2006) Transforming growth factor beta suppresses human telomerase reverse transcriptase (hTERT) by Smad3 interactions with c-Myc and the hTERT gene. J Biol Chem 281, 25588-25600

76. Horn S, Figl A, Rachakonda PS et al (2013) TERT promoter mutations in familial and sporadic melanoma. Science 339, 959-961

77. Huang FW, Hodis E, Xu MJ, Kryukov GV, Chin L and Garraway LA (2013) Highly recurrent TERT promoter mutations in human melanoma. Science 339, 957-959

78. Vinagre J, Almeida A, Populo $\mathrm{H}$ et al (2013) Frequency of TERT promoter mutations in human cancers. Nat Commun 4, 2185

79. Bell RJ, Rube HT, Kreig A et al (2015) Cancer. The transcription factor GABP selectively binds and activates the mutant TERT promoter in cancer. Science 348, 1036-1039

80. Griewank KG, Murali R, Puig-Butille JA et al (2014) TERT promoter mutation status as an independent prognostic factor in cutaneous melanoma. J Natl Cancer Inst 106, 1-13

81. Landa I, Ganly I, Chan TA et al (2013) Frequent somatic TERT promoter mutations in thyroid cancer: higher prevalence in advanced forms of the disease. J Clin Endocrinol Metab 98, E1562-1566

82. Liu X, Qu S, Liu R et al (2014) TERT promoter mutations and their association with BRAF V600E mutation and aggressive clinicopathological characteristics of thyroid cancer. J Clin Endocrinol Metab 99, E1130-1136

83. Liu R and Xing M (2014) Diagnostic and prognostic TERT promoter mutations in thyroid fine-needle aspiration biopsy. Endocr Relat Cancer 21, 825-830

84. Melo M, da Rocha AG, Vinagre J et al (2014) TERT promoter mutations are a major indicator of poor outcome in differentiated thyroid carcinomas. J Clin Endocrinol Metab 99, E754-765

85. George JR, Henderson YC, Williams MD et al (2015) Association of TERT Promoter Mutation, But Not BRAF Mutation, With Increased Mortality in PTC. J Clin Endocrinol Metab 100, E1550-1559

86. Pestana A, Vinagre J, Sobrinho-Simoes $M$ and Soares $P$ (2017) TERT biology and function in cancer: beyond immortalisation. J Mol Endocrinol 58, R129-R146

87. Wang K, Liu T, Ge N et al (2014) TERT promoter mutations are associated with distant metastases in upper tract urothelial carcinomas and serve as urinary biomarkers detected by a sensitive castPCR. Oncotarget 5, 1242812439

88. Liu W, Yin Y, Wang J et al (2017) Kras mutations increase telomerase activity and targeting telomerase is a promising therapeutic strategy for Kras-mutant NSCLC. Oncotarget 8, 179-190

89. Liu R, Zhang T, Zhu G and Xing M (2018) Regulation of mutant TERT by BRAF V600E/MAP kinase pathway through FOS/GABP in human cancer. Nat Commun 9, 579

90. Low KC and Tergaonkar V (2013) Telomerase: central regulator of all of the hallmarks of cancer. Trends Biochem Sci 38, 426-434

91. Yuan X, Larsson C and Xu D (2019) Mechanisms underlying the activation of TERT transcription and telomerase activity in human cancer: old actors and new players. Oncogene 38, 6172-6183

92. Zhou J, Ding D, Wang M and Cong YS (2014) Telomerase reverse transcriptase in the regulation of gene expression. BMB Rep 47, 8-14

93. Jafri MA, Ansari SA, Alqahtani MH and Shay JW (2016) Roles of telomeres and telomerase in cancer, and advances in telomerase-targeted therapies. Genome Med 8, 69

94. Marian CO, Cho SK, McEllin BM et al (2010) The telomerase antagonist, imetelstat, efficiently targets glioblastoma tumor-initiating cells leading to decreased proliferation and tumor growth. Clin Cancer Res 16, 154-163

95. Zanetti M (2017) A second chance for telomerase reverse transcriptase in anticancer immunotherapy. Nat Rev Clin Oncol 14, 115-128 